\title{
The Host Galaxies of High-Redshift Type Ia Supernovae
}

\author{
Mark Sullivan ${ }^{1}$, Richard Ellis $^{2}$, and the Supernova Cosmology Project ${ }^{3}$ \\ 1 University of Durham, South Road, Durham, DH1 3LE, UK; \\ mark. sullivan@durham.ac.uk \\ 2 105-24 Astronomy, Caltech, Pasadena, CA 91152, USA; \\ rse@astro.caltech.edu \\ ${ }^{3}$ saul@lbl.gov ${ }^{\star \star}$
}

Summary. We present new results on the Hubble diagram of distant type Ia supernovae (SNe Ia) segregated according to the type of host galaxy. This makes it possible to check earlier evidence for a cosmological constant by explicitly comparing SNe residing in galaxies likely to contain negligible dust with the larger sample. The cosmological parameters derived from these SNe Ia hosted by presumed dustfree early-type galaxies support earlier claims for a cosmological constant, which we demonstrate at $\simeq 5 \sigma$ significance, and the internal extinction implied is small even for late-type systems $\left(A_{B}<0.2\right)$. Furthermore, the scatter observed in the SNe Ia Hubble diagrams correlates closely with host galaxy morphology. We find this scatter is smallest for SNe Ia occurring in early-type hosts and largest for those in late-type galaxies. Moreover, SNe residing in late-type hosts appear fainter in their light-curve-width-corrected luminosity than those in early-type hosts, as expected if a modest amount of dust extinction is a contributing factor. Thus, our data demonstrate that host galaxy extinction is unlikely to systematically dim distant $\mathrm{SNe}$ Ia in a manner that would produce a spurious cosmological constant.

\section{Introduction}

Type Ia supernovae (SNe Ia) have emerged as important probes of the cosmological world-model. Empirical relationships between the peak intrinsic luminosity and the light-curve decay time can reduce the dispersion in their photometric properties to $\simeq 0.17 \mathrm{mag}$, making them excellent "calibrated candles" over a wide range in redshift. Systematic detection and calibration of high redshift $\mathrm{SNe}$ Ia over the last 10 years has led to two independent SN Ia Hubble diagrams, published by the Supernova Cosmology Project [3] (SCP) and the High-Redshift Supernova Search Team [6]. Data from both teams convincingly reject the deceleration expected from an Einstein-de Sitter (EdS) Universe, and provide evidence for a cosmic acceleration in a low mass-density Universe consistent with a non-zero vacuum energy density.

Such important results demand excellent supporting evidence, examining the homogeneity, environmental trends and evolutionary behavior of SNe Ia. Systematic differences in the peak magnitudes between high and low-redshift

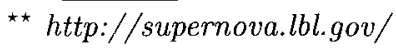


Table 1. The best-fitting cosmologies for SNe residing in each host galaxy type

\begin{tabular}{lcccccc}
\hline SNe Subset & Best $\Omega_{\Lambda}$ & $\begin{array}{c}\text { Mean } \\
\text { dispersion }\end{array}$ & DOF & $\begin{array}{c}\chi^{2} \\
/ \text { DOF }\end{array}$ & \multicolumn{2}{c}{$\begin{array}{c}\text { Probability that: } \\
\Omega_{\Lambda}>0\end{array}$} \\
& & $\Omega_{\Lambda} \geq \frac{\Omega_{\mathrm{M}}}{2}$ \\
\hline All SNe & $0.72_{-0.08}^{+0.09}$ & 0.199 & 51 & 1.121 & 99.79 & 99.59 \\
E/S0 & $0.57(0.58)_{-0.11}^{+0.12}$ & 0.159 & 12 & 0.680 & 97.09 & 94.80 \\
Spirals & $0.67(0.66)_{-0.06}^{+0.07}$ & 0.235 & 46 & 1.590 & 99.85 & 99.75 \\
$\quad$ Early & $0.50(0.50)_{-0.11}^{+0.12}$ & 0.200 & 22 & 1.309 & 92.46 & 86.83 \\
Late & $0.77(0.78)_{-0.07}^{+0.08}$ & 0.272 & 23 & 1.761 & 99.98 & 99.97 \\
\hline
\end{tabular}

SNe, arising either from evolutionary effects in the SNe progenitors or via subtle differences in the environments of the low and high-redshift galaxies in which SNe are produced, could mimic the cosmic acceleration without necessarily destroying the small dispersion seen in existing Hubble diagrams. Here, we present the results of a study examining the dependence of SN properties on host galaxy morphology via Hubble Space Telescope (HST) imaging and Keck spectroscopy. Disc and spheroidal stellar populations represent different star-formation histories, metallicities and, most importantly, dust content, and we might expect that SNe Ia progenitor composition and peak magnitudes/light-curve properties could be affected accordingly. In particular, we investigate how existing Hubble diagrams are affected by SNe lying in different types of host galaxy where the dust content of the hosts varies from type to type, and thus examine the effect of host galaxy extinction on the determination of the cosmological parameters.

\section{Datasets}

In this analysis we use the high-redshift SNe sample taken from the SCP [3]. We have three diagnostics available for the high-redshift sample: i) HSTSTIS/WFPC2 imaging providing morphology and SN location within the host, ii) Keck-II/ESI spectroscopy addressing the star-formation characteristics of the host galaxies, and iii) Ground-based $R, I$ imaging from the original SN discovery campaigns which acts as a consistency check on the other two diagnostics. We classify each host as either a spheroidal (E/S0), an early-type spiral (Sa-Sbc), or a late-type spiral/irregular (Scd and later types). In total, $93 \%$ of the high-redshift SNe sample can be classified using these data. We combine this high-redshift sample with existing low-redshift samples $[1,5]$ using published host galaxy classifications [2].

\section{Main Results}

The Hubble diagram for the high-redshift SNe Ia as a function of host galaxy type can be found in Fig. 1, and the results of the cosmological fits for the 


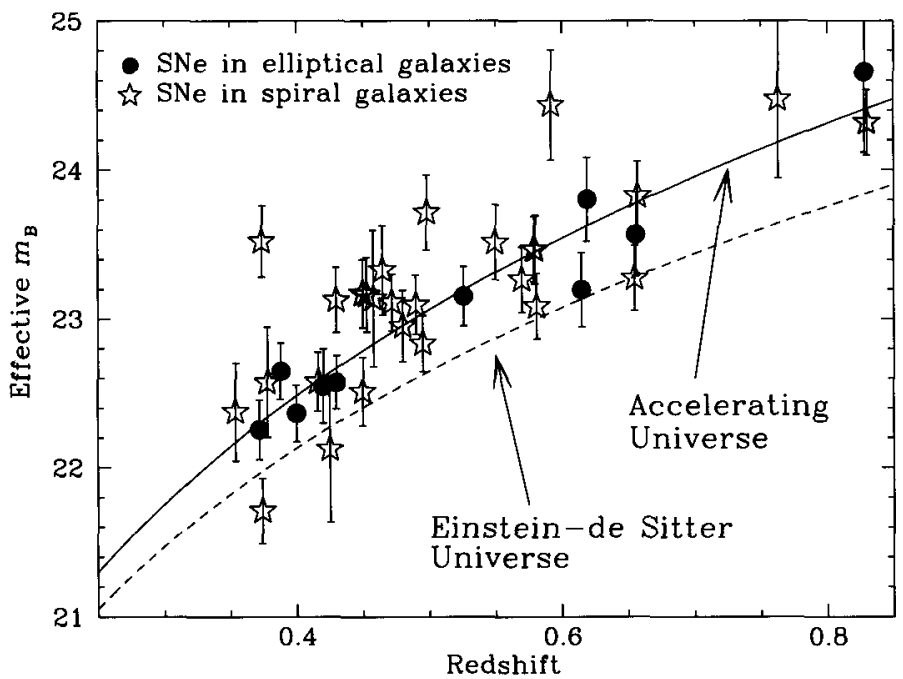

Fig. 1. The stretch-corrected high-redshift SNe Ia Hubble diagram plotted according to the class of the host galaxy. The expected brightness in both EdS and accelerating Universes are shown.

different SNe sub-classes are listed in Table 1 (see [7] for the full set of results). The principle finding is that all of the SNe subgroups strongly support cosmologies with $\Omega_{A}>0$, and the best-fitting cosmological models for each SN sub-group are broadly consistent within the error-bars.

Furthermore, $\mathrm{SNe}$ residing in $\mathrm{E} / \mathrm{S} 0$ galaxies provide 'tighter' cosmological fits, with smaller dispersions and $\chi^{2} /$ DOFs than the fits for SNe residing in later-type galaxies. The likely explanation for this result is that elliptical galaxies form a very homogeneous class of objects, being regular and usually dust-free when compared to the typically dusty nature of later-type galaxies. This hypothesis allows us to estimate the intrinsic spread in the peak magnitude of $\mathrm{E} / \mathrm{S} 0 \mathrm{SNe}$ compared to those residing in other classes of galaxy. Currently, each SNe error contains a contribution of 0.17 mag added in quadrature to allow for the uncertainty in the light-curve-width-luminosity correction, regardless of the host class. As some of this dispersion arises from host galaxy extinction, we can use the (presumed) dust-free E/SO SNe population to estimate the intrinsic dispersion for this class of SNe. For SNe in each host type, we therefore repeat the cosmological fits, altering the intrinsic dispersion until the $\chi^{2} /$ DOF for each fit is equal to one.

The intrinsic dispersions are (in magnitudes) $0.10,0.25$, and 0.24 for E/S0, early and late-type spirals/irregulars respectively. This small intrinsic dispersion seen for $\mathrm{SNe}$ in $\mathrm{E} / \mathrm{SO}$ matches very closely the dispersion seen in low-redshift $\mathrm{SNe}$ after a technique correcting for the effects of reddening has been applied (0.11 mag) [4]. The implication from this small dataset is clear: 


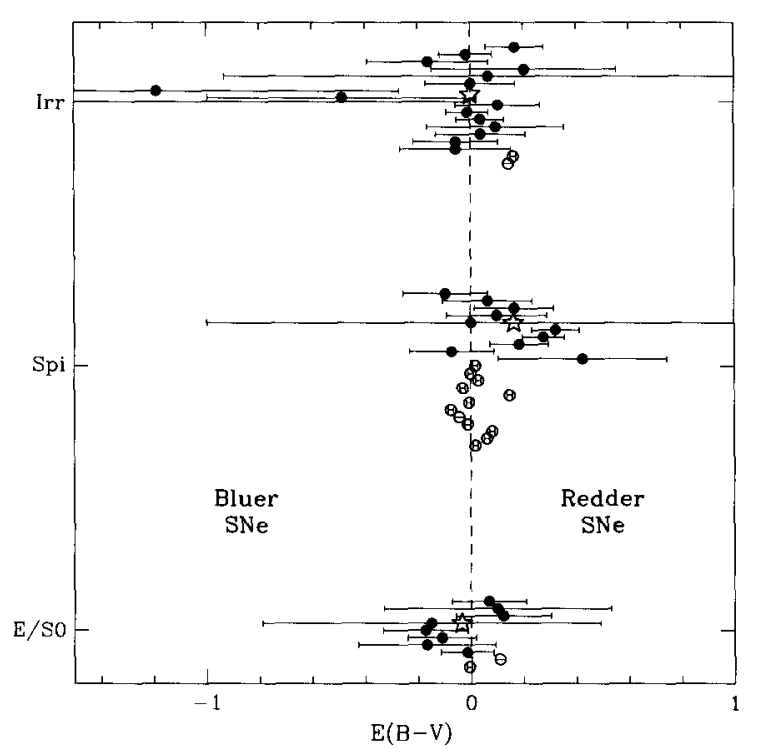

Fig. 2. The SN rest-frame "color excess" $(E(B-V))$ plotted as a function of the host galaxy type. The error-weighted mean of each distribution are shown as stars. The vertical offsets for each plotted SN is used to avoid over-plotting the data points and is illustrative only. High-redshift SNe are shown as filled circles, low-redshift $\mathrm{SNe}$ as open circles.

$\mathrm{SNe}$ residing in E/S0 hosts are superior "standard candles" than those in later-type galaxies due to the small amount of extinction affecting them.

We can further investigate the important issue of dust extinction on the SNe sample in two ways. Firstly, we can search for any differential extinction between the low and high-redshift samples by examining the distribution of the color excesses of the $\mathrm{SNe}$ (see $[3,7]$ for further details on how the color excess is measured), with larger color excesses representing redder $\mathrm{SNe}$ and hence likely higher extinctions. The distributions of the color excess at low and high-redshift are shown in Fig. 2, and appear very similar, with no clear offsets between the two populations. The mean color excess in $\mathrm{E} / \mathrm{S} 0$ galaxies is $\simeq 0$, indicating that this population is largely dust-free. In spiral galaxies the color excess is more significant at $\simeq 0.07$ - but this value is dominated by two red SNe which are excluded from the principle cosmological fit of [3]. When these two SNe are removed, the color excess drops to 0.03 in spiral galaxies [7].

The second test on the effect of dust on the SNe properties investigates the dust content of spiral galaxies compared to E/S0 galaxies. When using SNe Ia as standard candles, an intrinsic absolute magnitude for the SNe is fit for - but this intrinsic magnitude contains not just information on the 
brightness of the SN, but also information about the environment of each $\mathrm{SN}$, in particular the amount of dust that may be dimming the SN light. To investigate this, we re-fit the cosmologies for SNe in the different sub-groups, but this time fitting only for this intrinsic SN magnitude, and holding the cosmology fixed at the values derived for $\mathrm{SNe}$ in $\mathrm{E} / \mathrm{S} 0$ galaxies.

We find only a small difference between the brightness of $\mathrm{SNe}$ in E/S0s and spirals of $0.07 \pm 0.08$ magnitudes - the largest difference is between $\mathrm{E} / \mathrm{S} 0$ and late-type spirals at $0.14 \pm 0.09$. These values are only marginally significant, and provide little evidence for an increased reddening between $\mathrm{SNe}$ in E/S0 and spiral galaxies.

\section{Summary}

We have investigated the Hubble diagram of SNe Ia by classifying 39 highredshift events according to the their host galaxies as revealed by HST imaging, intermediate dispersion spectroscopy and broad-band colors. Together with morphological data for the host galaxies of local SNe drawn from the literature, we find the following.

1. SNe in all galaxy sub-groups provide convincing evidence for $\Omega_{\Lambda}>0$, though the scatter on the Hubble diagram does correlate closely with the host galaxy type. This scatter is minimal for SNe occurring in host galaxies classed as early-type, and increases towards later classes. The correlation does not depend on whether the SN are corrected for differences in light-curve shape.

2. We compare the color excesses of distant SNeIa with those observed locally and see no evidence for an increased extinction at higher-redshift. We see slight evidence for a type-dependent color excess, with, on average, spirals hosting the redder SNe.

3. SNe occurring in the later types are on average only marginally fainter than those in $\mathrm{E} / \mathrm{S} 0$ galaxies, with a difference in the absolute peak luminosities between $\mathrm{SNe}$ in late-type spirals and E/S0s of only $0.14 \pm$ $0.09 \mathrm{mag}$. This drops to $0.07 \pm 0.08 \mathrm{mag}$ when the spirals are taken as a whole.

We interpret these results according to the hypothesis that dust extinction in the host galaxy is the significant cause of the scatter in the Hubble diagram. Most importantly, the Hubble diagram confined to SNe Ia occurring in early-type host galaxies with presumed minimal internal extinction presents a very tight relationship and provides $\mathrm{a} \simeq 5 \sigma$ confirmation of a non-zero cosmological constant, assuming a flat Universe. When no assumption about the flatness of the Universe is made, $\mathrm{SNe}$ in $\mathrm{E} / \mathrm{S} 0$ galaxies imply $\Omega_{\Lambda}>0$ at nearly 98 per cent probability. Furthermore, the trends in extinction by host galaxy morphology and between low and high-redshift examples implies only 
modest extinctions in spirals, though with a large galaxy-to-galaxy scatter. Our study is a good illustration of how ancillary data on host galaxies can be used to examine the nature and reliability of SNe as probes of the cosmological expansion history. We show that internal dust extinction cannot be a primary contaminant and that SNe Ia, particularly those occurring in spheroidal galaxies which can be readily screened with spectroscopic, colors and morphological data, represent a very powerful cosmological probe.

\section{References}

1. M. Hamuy et al. : Astron. J. 112, 2408 (1996)

2. M. Hamuy et al. : Astron. J. 120, 1479 (2000)

3. S. Perlmutter et al. : Astrophys. J. 517, 565 (1999)

4. M.M. Phillips et al. : Astron. J. 118, 1766 (1999)

5. A.G. Riess et al. : Astron. J. 117, 707 (1999)

6. B.P. Schmidt et al. : Astrophys. J. 507, 46 (1998)

7. M. Sullivan et al. : Mon. Not. R. Astron. Soc. 340, 1057 (2003) 